Original article

\title{
Trypanosoma cruzi infection alters glucose metabolism at rest and during exercise without modifying the morphology of pancreatic islets in rats
}

\author{
Rômulo D. Novaes ${ }^{a}$, Reggiani V. Gonçalves ${ }^{b}$, Arlete R. Penitente ${ }^{a}$, André Talvani $^{c}$, Clóvis A. Neves $^{a}$, \\ Antônio J. Natali ${ }^{\mathrm{d}}$, Izabel R.S.C. Maldonado ${ }^{\mathrm{a}, *}$ \\ a Department of General Biology, Federal University of Viçosa, Viçosa, Minas Gerais, Brazil \\ ${ }^{\mathrm{b}}$ Department of Morphology, Federal University of Pelotas, Pelotas, Rio Grande do Sul, Brazil

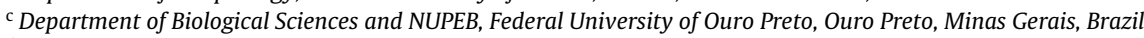 \\ ${ }^{\mathrm{d}}$ Department of Physical Education, Federal University of Viçosa, Viçosa, Minas Gerais, Brazil
}

\section{A R T I C L E I N F O}

\section{Article history:}

Received 6 December 2011

Received in revised form 26 April 2012

Accepted 24 May 2012

\section{Keywords:}

Chagas' disease

Glucose metabolism

Pancreas

Pathology

Exercise

\begin{abstract}
A B S T R A C T
This study investigated the effects of Trypanosoma cruzi infection on pancreatic morphology and glucose metabolism at rest and during exercise. Wistar rats were randomized into control $(C G=10)$ and infected $(\mathrm{IG}=10)$ groups. The IG animals were inoculated with $T$. cruzi Y strain (300,000 trypomastigotes/50 g). After 9 weeks, the animals were subjected to glucose (OGTT) and insulin (ITT) tolerance tests and a treadmill running protocol. Blood glucose, lactate and time to fatigue were determined. After euthanasia, the pancreases were removed for morphological and biochemical analyses. The IG presented abnormal glucose kinetics in OGTT and a similar glucose curve in ITT compared to the CG. During the exercise test, the IG showed anticipation of time to fatigue. At the point of fatigue, no difference was found in blood glucose and lactate between the groups. There was a significant correlation between lactate levels and the time to fatigue. The IG presented marked pancreatic inflammation, fibrosis and protein oxidation. The number of $\beta$ cells in the IG animals was not reduced. T. cruzi infection impaired pancreas morphology and glucose metabolism at rest and during exercise in rats, which could constitute an additional mechanism in the induction of exercise intolerance in Chagas' disease.
\end{abstract}

(c) 2012 Elsevier GmbH. All rights reserved.

\section{Introduction}

Chagas' disease (ChD) is a neglected illness caused by the intracellular protozoan parasite Trypanosoma cruzi (T. cruzi), which remains as an important health problem in 18 developing countries in South and Central America [4,28]. Its main clinical manifestations are cardiac and/or digestive disturbances, with a prevalence of about 12-14 million cases worldwide [28].

Although there is sufficient evidence showing that $T$. cruzi is able to spread and infect several organs such as the gonads, kidney, liver and pancreas in humans [30,32] and animals [7,33,34], these infection sites have not been researched to the same extent as cardiac and intestinal manifestations, and thus remain neglected. Some studies addressed the involvement of the pancreas in $\mathrm{ChD}$ [7,30,32-34]. Traditionally, this observation has been based on evidence of altered glucose and insulin responses after oral glucose tolerance tests in chronic chagasic patients and post-mortem

\footnotetext{
* Corresponding author at: Universidade Federal de Viçosa, Departamento de Biologia Geral, Edifício Chotaro Shimoya, Avenida PH Rolfs, s/n, CEP: 35570-000 Viçosa, Minas Gerais, MG, Brazil. Tel.: +55 313899 3359; fax: +55 3138992549.

E-mail address: irscosta@ufv.br (I.R.S.C. Maldonado).
}

histopathological analyses of the pancreas [18,35,37]. Although the direct pathological and functional repercussions of $T$. cruzi on the heart and gut are the primary focus of investigations and interventions in ChD [4,28], it is evident that pancreatic infections also present an important clinical significance in this disease [26,35]. This importance is clear according to the concept of the enteroinsular axis, considering the interrelationships of pancreatic secretions and the intestinal tube in several peptidergic routes that participate in the metabolism of energy substrates [8]. In fact, it has been shown that patients with the digestive form of chronic Chagas' disease frequently present variable degrees of pancreatic morphological changes and glucose metabolism dysfunctions [18,26]. In addition, previous studies suggested that chagasic individuals present permanently elevated blood glucose levels and a high predisposition toward the development of type 2 diabetes mellitus (DM2) compared to healthy subjects $[18,26,35]$.

Glucose is an important compound related to exercise performance under healthy and diseased conditions [37]. Clinical investigations have shown that chagasic subjects present a reduced functional capacity and exercise tolerance $[10,16,17,20]$. However, few studies have evaluated the factors that affect the capacity to perform physical exercise in patients with $\mathrm{ChD}$, and exercise intolerance has primarily been associated with changes in the 
mechanical and electrical activities of cardiac and skeletal muscle tissues $[10,16,17,20,23]$. Currently, several aspects of the pathogenesis of exercise intolerance in ChD still need to be clarified. To the best of our knowledge, up to the present date no studies on humans or animal models have been carried out to investigate blood glucose levels during exercise in $T$. cruzi infection. Thus, the present study was designed to investigate the effects of $T$. cruzi infection on pancreatic morphology and glucose metabolism at rest and during exercise and the relationship between these variables and exercise tolerance. We hypothesized that the dysfunction in glucose metabolism might be an additional component involved in reduced exercise tolerance in rats.

\section{Materials and methods}

\section{Animals and infection}

Twenty 4-month-old male Wistar rats (Rattus norvegicus) with an initial weight of $366.25 \pm 31.17 \mathrm{~g}$ were used in the experiments and were provided with a rodent diet (AIN-93M) [29] and water ad libitum. The animals were divided into an uninfected control group (CG, $n=10$ ) and an infected group (IG, $n=10$ ) and maintained under a controlled environment with a temperature of $22 \pm 2{ }^{\circ} \mathrm{C}$ and $12 \mathrm{~h}$ light/dark cycles. The rats of the IG were inoculated intraperitoneally with $1 \mathrm{~mL}$ of infected mice blood containing the $T$. cruzi Y strain (300,000 trypomastigotes $/ 50 \mathrm{~g}$ body weight), according to the method of Martinelli et al. [22]. Infection was confirmed by the presence of trypomastigotes in peripheral blood aliquots collected 4 days after inoculation [3]. All animals were euthanized 9 weeks after inoculation by cervical dislocation while under anesthesia. The experimental protocols were conducted in accordance with the Brazilian College for Animal Experimentation and were approved by the Animal Research Ethics Committee of the Federal University of Viçosa, Brazil (protocol 30/2009).

\section{Body mass and underweight}

Before being euthanized, the height and weight of the animals were measured to determine the body mass and calculate the Lee index. The Lee index is an indicator of underweight, estimated by the formula $\sqrt[3]{\text { body mass }(\mathrm{g})} /$ snout-anus length $(\mathrm{cm})[1]$.

\section{Pancreatic function and insulin tolerance}

The oral glucose tolerance test (OGTT) was used to investigate pancreatic function through the insulin response induced by a glycemic load. Prior to the OGTT, all rats were fasted for $16 \mathrm{~h}$, although water was not restricted. Then, anhydrous glucose was orally administered to the animals ( $3 \mathrm{~g} / \mathrm{kg}$ of body weight) diluted in $3 \mathrm{~mL}$ distilled water $(\mathrm{w} / \mathrm{v})$ with a feeding syringe. Blood samples were collected by tail vein puncture at 0 (fasting glucose), 30, 60, 90 and $120 \mathrm{~min}$ after the glucose load to determine blood glucose via the glucose oxidase method (OneTouch Ultra ${ }^{\circledR}$, Johnson \& Johnson, CA, USA). The area under the blood glucose response curve (AUC) was calculated for each animal using the trapezoidal method [27].

Forty-eight hours after OGTT, the insulin tolerance test (ITT) was performed to investigate the peripheral tolerance to insulin. Before the ITT, the animals were fasted for $2 \mathrm{~h}$ to eliminate any differences in stomach contents or plasma insulin between the rats. The rats were injected subcutaneously at the back of the neck with 0.5 units/kg insulin (Biochemica, FL, USA), and the blood glucose was measured at the same times described for the OGTT $(0,30,60$, 90 and $120 \mathrm{~min}$ ) using the same glucose oxidase method. The ratio of the glucose decay constant $\left(K_{\text {ITT }}\right)$ was calculated using the formula $0.693 /\left(T_{1 / 2}\right)$, where the $T_{1 / 2}$ of plasma glucose was determined from the glucose curve during its phase of linear decay (0-30 min) [9].

\section{Exercise test protocol and metabolic parameters}

Each rat was subjected to an aerobic running test until fatigue $48 \mathrm{~h}$ after the ITT, according to the incremental running protocol described by Koch and Britton [14]. Prior to the exercise test, the rats were familiarized with the motor-driven treadmill (Insight Instruments ${ }^{\circledR}$, Ribeirão Preto, SP, Brazil) by running at a speed of $10 \mathrm{~m} / \mathrm{min}$ at a $5 \%$ inclination for $5 \mathrm{~min}$ per day for 7 consecutive days. This amount of exposure to treadmill running is slightly below that required to produce metabolic adaptations [14]. The test was performed at a constant slope of $5 \%$ with a starting speed of $10 \mathrm{~m} / \mathrm{min}$. The treadmill velocity was increased by $1 \mathrm{~m} / \mathrm{min}$ every $2 \mathrm{~min}$, and each rat was run until fatigued. Fatigue was defined as the point at which the animals were no longer able to keep pace with the treadmill [15]. Immediately before and after the test, the blood levels of lactate and glucose were measured using reflectance photometry (Accutrend Lactate ${ }^{\circledR}$, Roche, Basel, Switzerland) and glucose oxidase (OneTouch Ultra ${ }^{\circledR}$, Johnson \& Johnson, CA, USA), respectively. The distance traveled $(\mathrm{m})$ and time to fatigue (min) were also recorded.

\section{Protein oxidation, fibrosis}

The protein carbonyl content was measured using the 2,4dinitrophenylhydrazine (DNPH) procedure [38]. Total protein levels in the lung tissue were measured using the Bradford method [2].

For each group, 20 sections of $8 \mu \mathrm{m}$ thickness stained with Sirius red and Fast green were used to quantify the amount of collagen and total protein in pancreatic tissue using a previously described spectrophotometric method [19]. In this method, the maximal absorbance of Sirius red $(540 \mathrm{~nm})$ and Fast green $(605 \mathrm{~nm})$ dyes corresponds to the amount of collagen and non-collagen proteins, respectively.

\section{Histological processing, histochemistry and immunohistochemistry}

After the animals were euthanized, each pancreas and liver were removed in totum and placed in histological fixative for $48 \mathrm{~h}$ (freshly prepared $10 \%(\mathrm{w} / \mathrm{v})$ formaldehyde in $0.1 \mathrm{M}$ phosphate buffer, $\mathrm{pH}$ 7.2). Pancreas and liver fragments were dehydrated in ethanol, cleared in xylol and embedded in paraffin. Blocks were cut into 4 and $8 \mu \mathrm{m}$ sections and mounted on histological slides. The pancreas sections were stained by hematoxylin-eosin (H\&E), Fast green and Sirius red for histopathological analysis and aldehyde-fuchsin [11] and indirect immunoperoxidase to localize the insulin-producing $\beta$ cells that were evaluated in the stereological and kariometric analysis. According to direct peroxidase technique [39], the sections were subjected to immunohistochemistry using anti-insulin monoclonal antibodies (lot number A90-117p-4, Bethyl Laboratory, Montgomery, TX, USA). The optimum dilution of the primary antibody was 1:500 and for the secondary antibody conjugated with peroxidase it was $1: 200$. The liver sections were stained with the best carmine method for glycogen [25]. Digital images were captured using a light microscope (Olympus BX-60 ${ }^{\circledR}$, Tóquio, Japan) connected to a digital camera (Olympus QColor- $3^{\circledR}$, Tokyo, Japan).

\section{Histopathology, stereology and karyometry}

The inflammatory process was evaluated by determining the relationship between the total number of polymorphonuclear $(\mathrm{PMN})$ and mononuclear (MN) cells observed in the pancreas of 

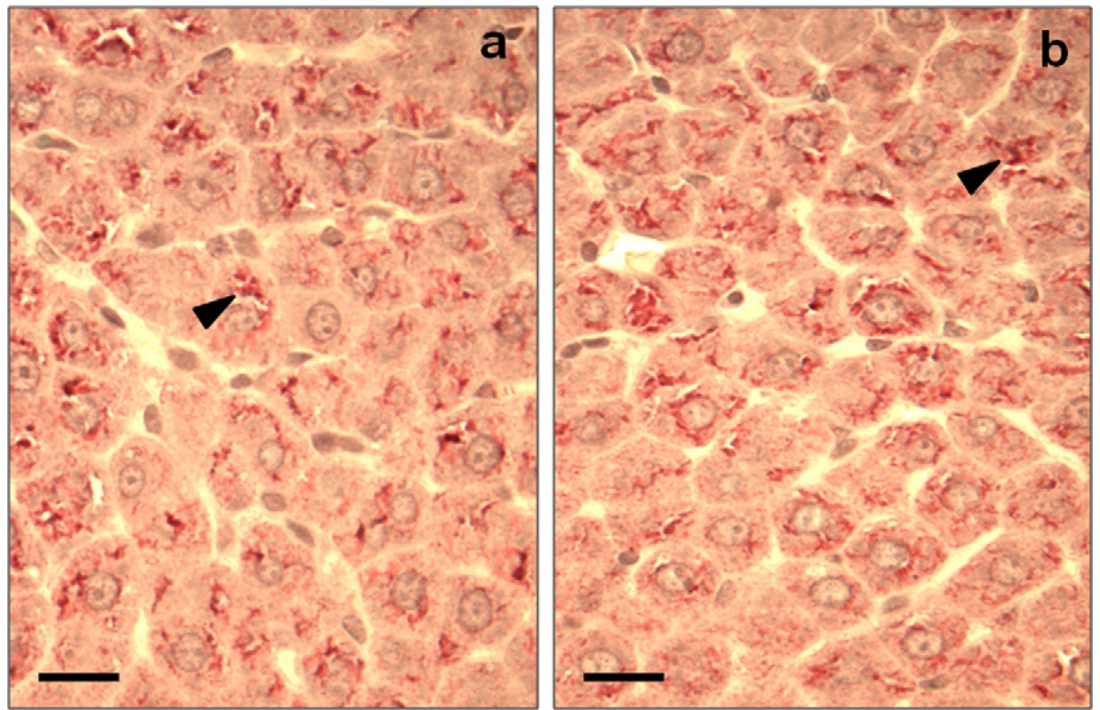

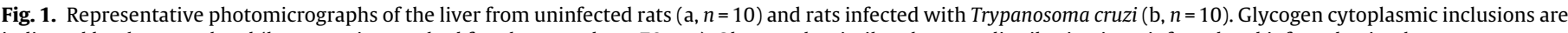
indicated by the arrowhead (best carmine method for glycogen; bar $=70 \mu \mathrm{m}$ ). Observe the similar glycogen distribution in uninfected and infected animals.

control and infected animals [5]. These cells were evaluated in a test area of $3.4 \times 10^{3} \mu \mathrm{m}^{2}$ at a magnification of $1000 \times$ across five random, non-coincident microscopic fields for each animal and a total of $170 \times 10^{3} \mu \mathrm{m}^{2}$ of pancreas tissue for each group [12]. All morphological analyses were performed using the image analysis software Image Pro-Plus $4.5^{\circledR}$ (Media Cybernetcs, Silver Spring, MD, USA).

The stereological parameters analyzed were: $(A)$ the volume density $(\mathrm{Vv})$ of the islets in pancreatic tissue $\left(\mathrm{Vv}_{\mathrm{i}}\right)$ and $\beta$ cells per islet $\left(\mathrm{Vv}_{\mathrm{C}}\right)$; (B) the number of $\beta$ cells per islet area $\left(N_{\mathrm{AC}}\right)$ and islet volume $\left(N_{\mathrm{VC}}\right)$; and $(\mathrm{C})$ the islet volume $\left(V_{\mathrm{i}}\right)$. The point-counting method was used to estimate the parameters of item A using the formula $\mathrm{Vv}_{\text {[structure }]}=P_{P[\text { structure }]} / P_{T}$, where $P_{P}$ is the number of points that hit the structure and $P_{T}$ is the total number of test points [21]. For this analysis, $\mathrm{Vv}_{\mathrm{i}}$ was estimated using a test system of 200 points in a standard test area of $73 \times 10^{3} \mu \mathrm{m}^{2}$ across five random, non-coincident microscopic fields for each animal and a total of $3.6 \times 10^{6} \mu \mathrm{m}^{2}$ of pancreas tissue for each group. The relationship between the number of points of the test system that hit the islet profile and those that specifically hit $\beta$ cells was used to calculate $\mathrm{Vv}_{\mathrm{c}}$. A total of 100 islet profiles of the pancreas were examined in order to estimate items $\mathrm{B}$ and $\mathrm{C}$. The $\mathrm{Vv}_{\mathrm{i}}$ was investigated under a magnification of $40 \times$, and the other parameters were determined under a magnification of $400 \times$.

The density of $\beta$ cells per islet profile $\left(N_{\mathrm{AC}}, \beta\right.$ cells $\left./ \mu \mathrm{m}^{2}\right)$ was calculated by dividing the number of nuclei per islet profile by the mean islet area. The nuclear profiles were counted using the direct counting method under a magnification of $200 \times$. The islet area $\left(A_{\mathrm{i}}\right)$ was measured directly using the image contour function of the Image Pro-Plus $4.5^{\circledR}$ software (Media Cybernetcs, Silver Spring, MD, USA). The numerical density of $\beta$ cells per unit volume of islet cells $\left(N_{\mathrm{VC}}, \beta\right.$ cells $/ \mu \mathrm{m}^{3}$ ) was determined using a physical dissector $(d)$ method. In this method, the distance between lookup and look-down sections $(t)$ was $3 \mu \mathrm{m}$ for each pair $d$. Thus, $\beta$ cells seen in lookup in anterior sections but not the look-down sections were counted $\left(Q_{\mathrm{n}}\right)$, and the numerical density of islets $\left(N_{\mathrm{VC}}\right)$ was estimated as: $N_{\mathrm{VC}}=Q_{\mathrm{n}} / A_{\mathrm{i}} \times t$, where $A_{\mathrm{i}}$ is the islet area observed in sections of thickness $t(3 \mu \mathrm{m})$. The absolute number of $\beta$ cells per islet ( $N, \beta$ cell/islet) was determined by multiplying $N_{\mathrm{Vc}}$ by the islet volume. The islet volume $\left(V_{\mathrm{i}}\right)$ was calculated using Cavalieri's principle: $V_{\mathrm{i}}=\Sigma t \times \Sigma A_{\mathrm{i}}[21]$.
Sections stained with aldehyde-fuchsin were used for the karyometric study of $\beta$-cell nuclei. In the karyometric analysis, $50 \beta$ cell nuclei for each animal were analyzed under a magnification of $400 \times$. Using a calibrated linear scale, the longest axis $(D)$ and shortest axis $(d)$ of the $\beta$ cells were measured. The geometric axis of the nucleus $\left(D_{\mathrm{n}}\right)$ was calculated using the equation $D_{\mathrm{n}}=(D \times d)^{1 / 2}$ This parameter was used to calculate the nuclear volume from the formula $V=\pi / 6 \times D_{\mathrm{n}}$ [31].

In liver sections stained with the carmine method, the volume density of glycogen cytoplasmic inclusions in the histological area ( $\mathrm{Vv}[g l y c], \%)$ was estimated according to a stereological protocol previously described [25].

\section{Statistical analysis}

The data are presented as the mean \pm standard deviation (mean \pm SD). Normal distribution of the data was verified using the Kolmogorov-Smirnov test. The blood glucose, lactate, protein carbonyl levels and index of inflammatory processes were compared using the Student's $t$-test. Stereological and karyometric data were compared using the Mann-Whitney $U$ test. The relationship between blood glucose, lactate and total time to fatigue was assessed by linear regression. A probability of $p<0.05$ was considered statistically significant.

\section{Results}

Body mass, underweight and liver glycogen

No statistical difference was found between the groups for body mass (CG, 502.17 $\pm 20.41 \mathrm{~g} v \mathrm{~s}$. IG, $494.69 \pm 31.06 \mathrm{~g}$ ), Lee index (CG $311.89 \pm 17.93 v s$. IG, $300.04 \pm 13.21$ ), or the volume density of glycogen inclusions in the liver tissue (CG, $16.38 \pm 4.03 \%$ vs. IG $17.10 \pm 5.57 \%$ ) (Fig. 1 ).

\section{Pancreatic functioning and insulin tolerance}

Animals of the IG presented fasted glucose levels that were significantly higher than the CG animals $(p<0.05)$. In the OGTT, the IG animals showed high levels of blood glucose at all testing times and abnormal glucose kinetics compared to the CG. For the IG animals, 
OGTT
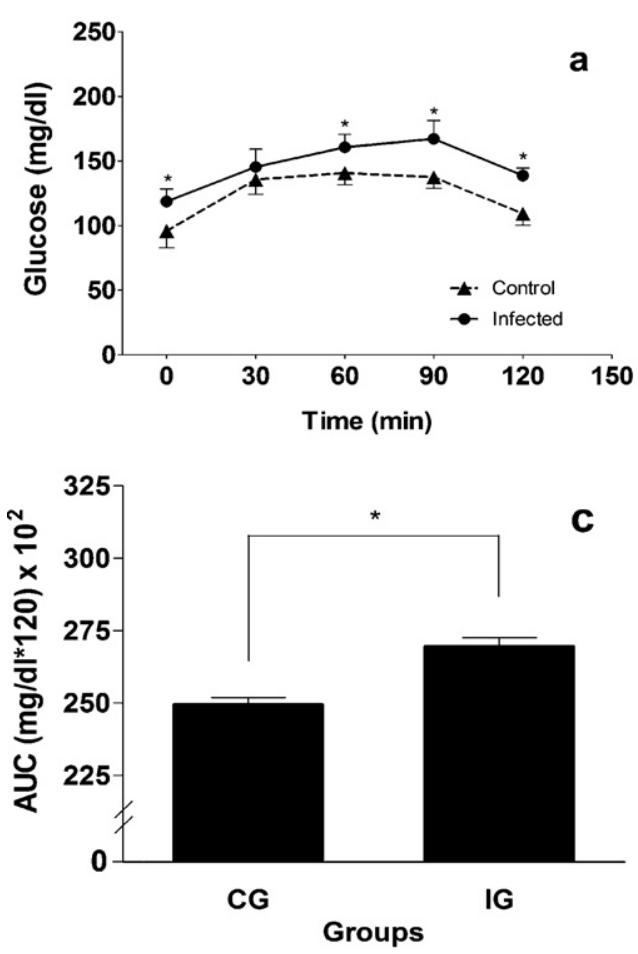

ITT
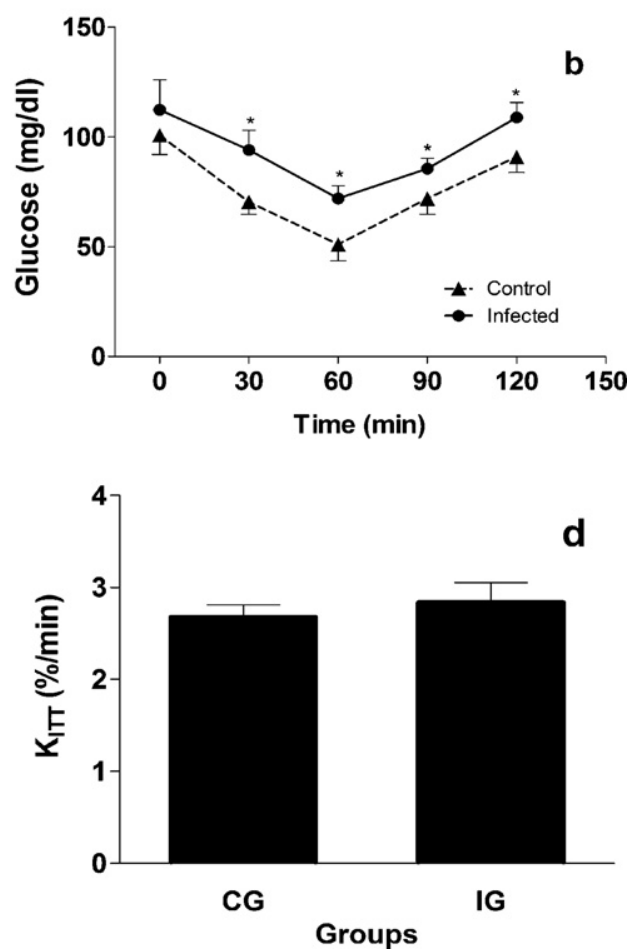

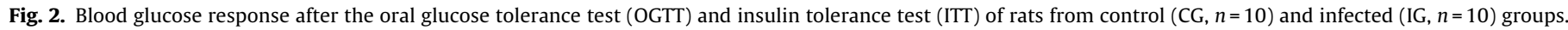

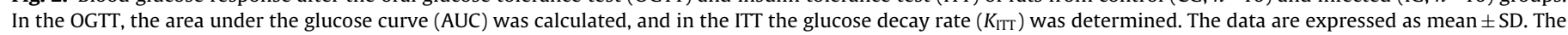
statistical difference between groups was ${ }^{*} p<0.001$.

the increase in blood glucose was prolonged and the glucose decay was delayed, only occurring after $90 \mathrm{~min}$ (Fig. 2a). The area under the glucose curve was significantly higher in the IG compared to the CG (Fig. 2c).

The levels of blood glucose in the ITT were higher in the IG animals during the test compared to the CG animals, with significant difference after $30 \mathrm{~min}$ (Fig. 2b). In addition, the glucose decay rate $\left(K_{\text {ITT }}\right)$ in this test was similar between the groups (Fig. $2 \mathrm{~d}$ ).

\section{Exercise test protocol and metabolic parameters}

Infection with T. cruzi impaired exercise tolerance, with a significant reduction in distance traveled and total time to fatigue compared to the CG (Fig. 3a and b). Before exercise, the IG animals presented blood glucose levels that were significantly higher compared to the CG. At this moment in time, there was no significant difference between the lactate levels in the IG and CG groups. At the point of fatigue, the blood glucose level decreased and the lactate level increased compared to the levels at the beginning of the exercise in both groups. However, no statistical differences were found between the groups for these variables at the point of fatigue (Fig. 3c and d). An inverse and significant correlation was found between the blood levels of lactate and glucose at the point of fatigue and between lactate and total time to fatigue during the exercise test (Fig. 4a-d) in both groups.

\section{Histopathological analysis}

Histopathological analysis of the heart confirmed the presence of cardiac infection by amastigote forms of $T$. cruzi with cardiomyocyte hypertrophy (Fig. 5b). At the same time, pancreatic inflammation was also evident, and vacuolization of acinar cells was observed along with evidence of cell apoptosis in the IG (Fig. $5 g-j$ ). In addition, marked inflammatory infiltrates were found in the exocrine pancreas of IG animals with a dominant perivascular, periductal and periacinar distribution (Fig. $5 \mathrm{~g}$ and $\mathrm{j}$ ). The infiltration of inflammatory cells into pancreatic islets was scarce (Fig. 5g, h and j).

The number of both MN and PMN cells was significantly higher in IG compared to CG $(p<0.05)$ (Fig. 6a). The IG animals also showed significantly higher collagen and protein carbonyl contents compared to CG animals $(p<0.05)$ (Fig. $6 \mathrm{~b}$ and $c)$.

\section{Stereology and karyometry}

The quantitative morphological parameters are shown in Table 1. No significant differences were found between the groups for all relative and absolute parameters estimated in the stereological analysis. The karyometric variables were significantly reduced in IG animals compared to CG animals $(p<0.05)$.

\section{Discussion}

In the present study, infection was induced with the T. cruzi Y strain due to its high virulence and capacity to parasitize several organs and tissues. Moreover, this strain has also shown evidence of a similar immune-pathogenic mechanism in humans and rats [24]. Due to the resistance of rats to $T$. cruzi, a high parasite number was used in the inoculum to induce infection, which was confirmed in all of the IG animals. Our results showed that the inoculum and the consequential T. cruzi infection were sufficient to induce a marked dysfunction in glucose kinetics at rest and during exercise and a reduction in exercise tolerance in the IG animals. Interestingly, such alterations occurred in the presence of marked morphological changes in the exocrine pancreas and minimal changes in the endocrine pancreas.

Changes in glucose metabolism in ChD have been reported in human [18,26,35] and animal model of $T$. cruzi infection [34]. 

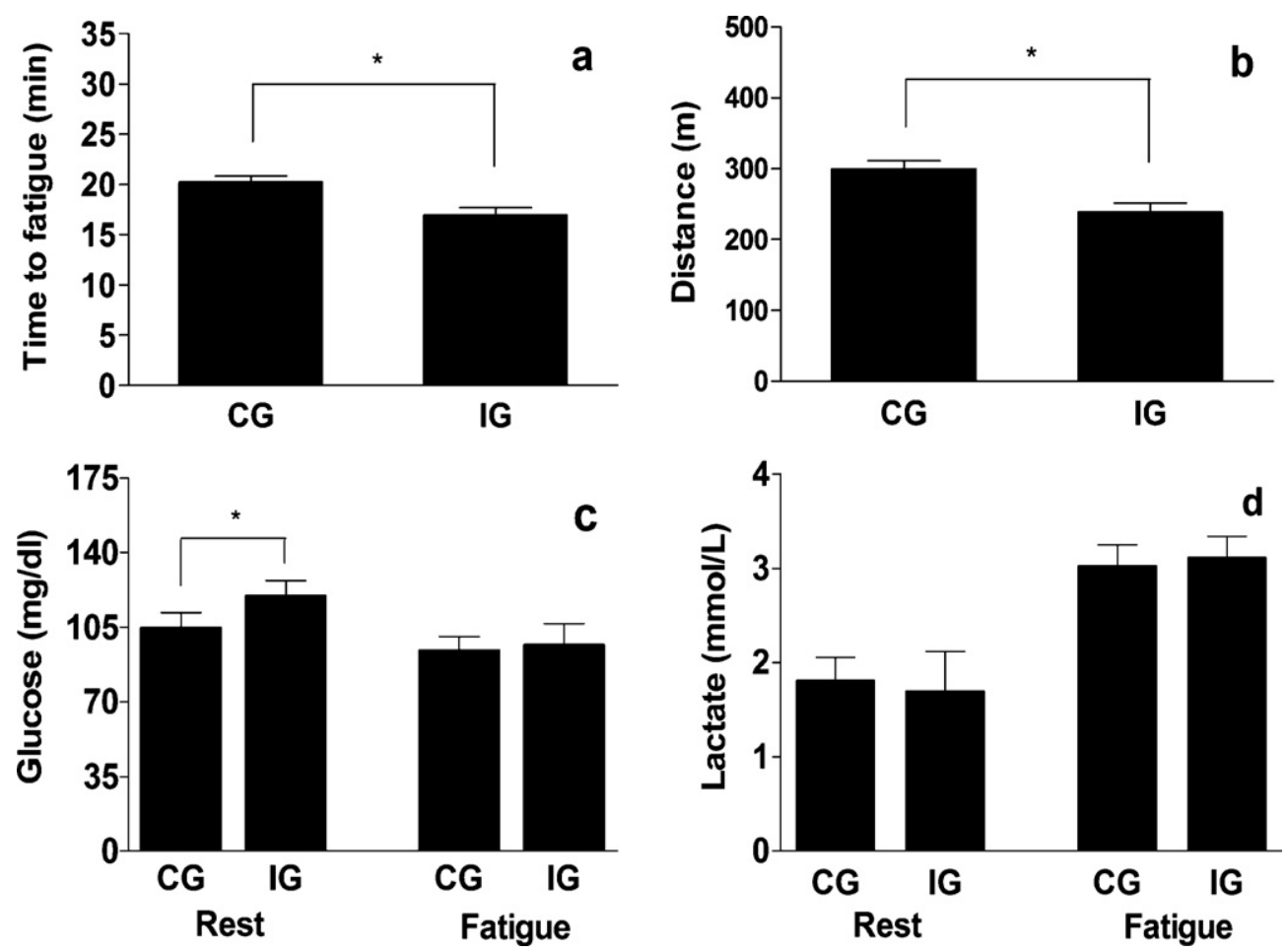

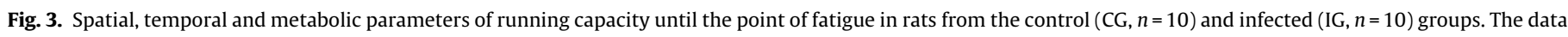
are expressed as mean \pm SD. The statistical difference between the groups was $* p<0.001$.

Evidence has been found showing that the modifications in body composition are able to alter the insulin response and consequently glucose kinetics [9]. Due to the systemic characteristics of $\mathrm{ChD}$, the occurrence of anorexia and weight loss are not unusual [36], which are events that can affect the levels of blood glucose in OGTT and ITT and during exercise. After 9 weeks of infection, no significant difference was found in body mass, Lee index, or the volume density of glycogen inclusions in the liver tissue between IG and CG animals, a finding that reduced the influence of body mass and changes in the glycogen stores on the results obtained.

Since the same glucose curves were found in the ITT for both the IG and CG animals, a dysfunction in glucose kinetics does not
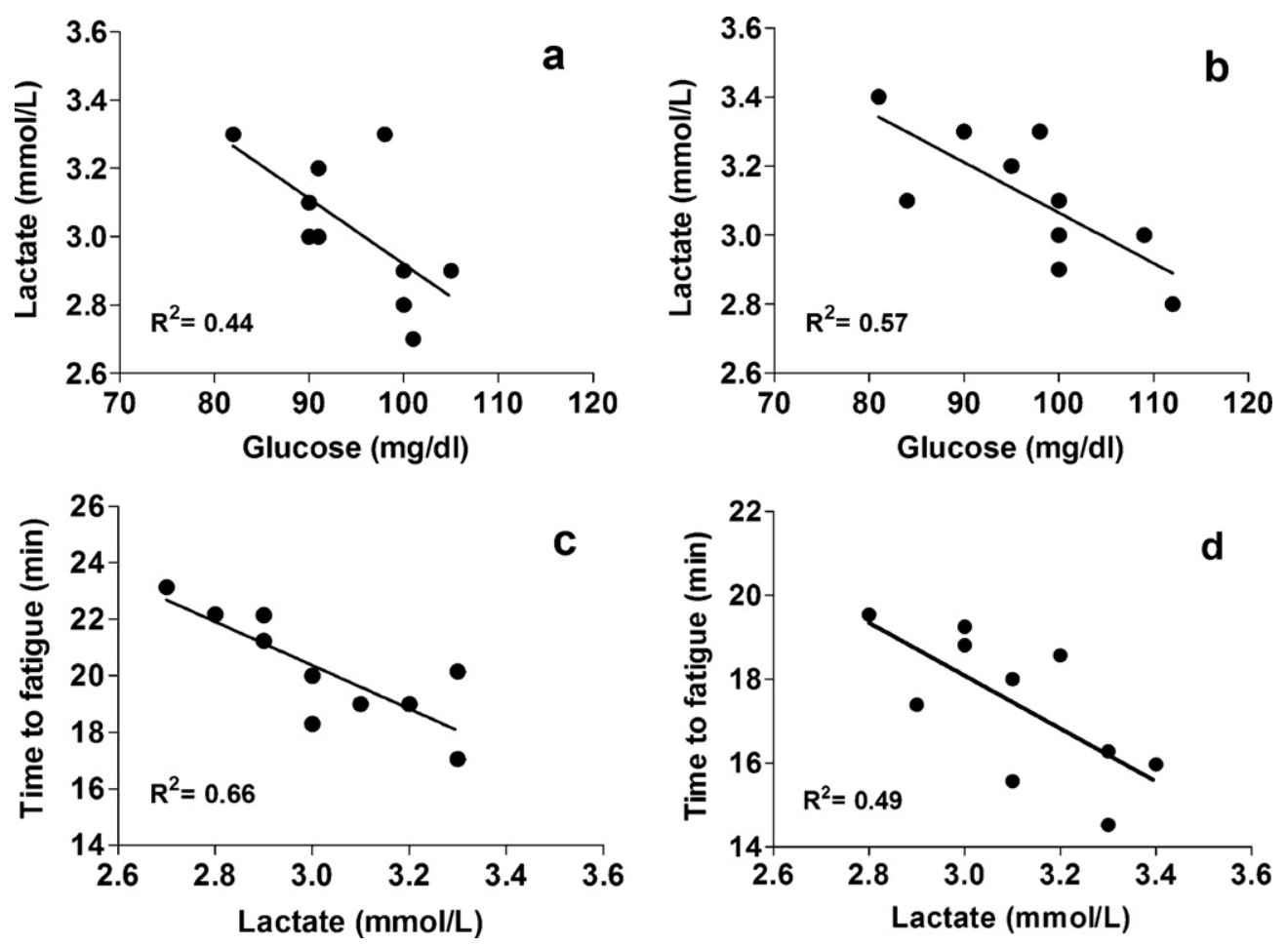

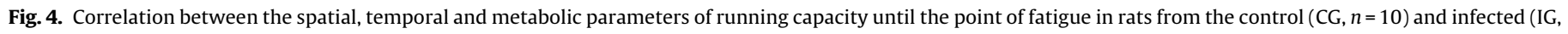

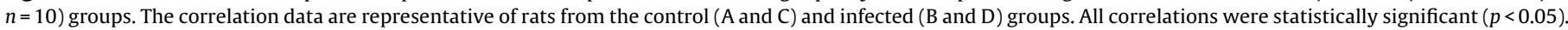



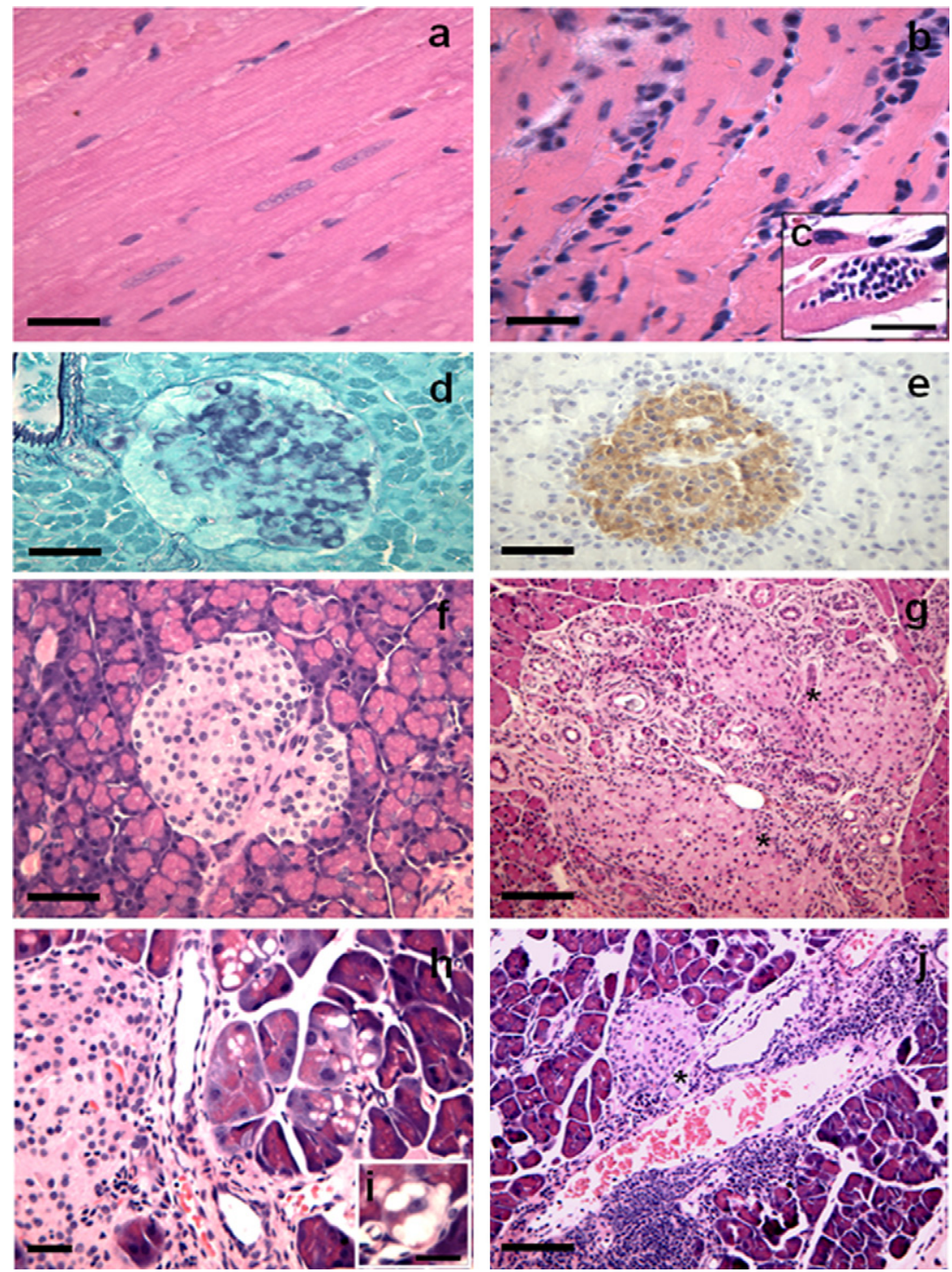

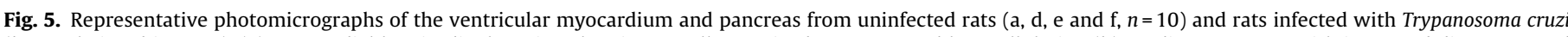

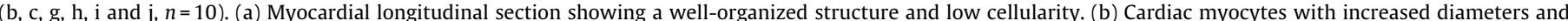

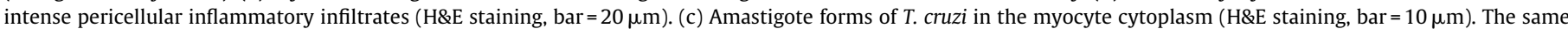

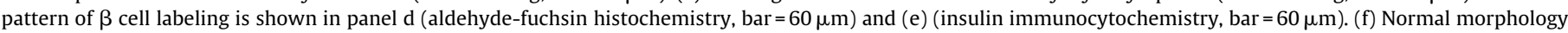

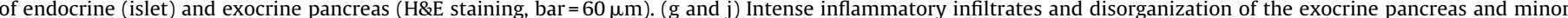

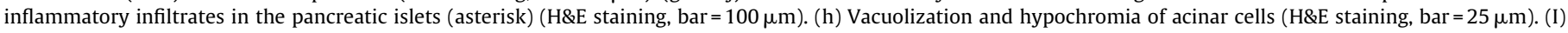
Detailed cell vacuolization, suggesting the occurrence of apoptosis (H\&E staining, bar $=15 \mu \mathrm{m}$ ).

Table 1

Stereology of the pancreatic tissue and karyometry of $\beta$ cell nuclei in rats from control (CG, $n=10$ ) and infected (IG, $n=10$ ) groups.

\begin{tabular}{|c|c|c|}
\hline Parameters & Control group & Infected group \\
\hline Vv [islet] (\%) & $2.920 \pm 1.451$ & $2.760 \pm 1.370$ \\
\hline$V_{\mathrm{i}}\left[\mu \mathrm{m}^{3}\right]$ & $2,022,648.264 \pm 641,182.222$ & $1,727,643.212 \pm 759,538.311$ \\
\hline $\mathrm{Vv}[\beta$ cell $](\%)$ & $64.690 \pm 10.950$ & $60.480 \pm 9.300$ \\
\hline$N_{\mathrm{AC}}\left[\beta\right.$ cells $\left./ \mu \mathrm{m}^{2}\right]$ & $0.010 \pm 0.005$ & $0.008 \pm 0.003$ \\
\hline$N_{\mathrm{Vc}}\left[\beta\right.$ cells $\left./ \mu \mathrm{m}^{3}\right]$ & $0.043 \pm 0.014$ & $0.034 \pm 0.010$ \\
\hline$N[\beta$ cell $/$ islet $]$ & $69,250.763 \pm 28,165.934$ & $73,145.215 \pm 36,424.107$ \\
\hline Nuclei geometric axis $(\mu \mathrm{m})$ & $7.130 \pm 0.530$ & $6.460 \pm 0.630^{*}$ \\
\hline Nuclei area $\left(\mu \mathrm{m}^{2}\right)$ & $12.770 \pm 1.930$ & $10.520 \pm 1.990^{*}$ \\
\hline Nuclei volume $\left(\mu \mathrm{m}^{3}\right)$ & $192.650 \pm 44.480$ & $144.790 \pm 39.820^{*}$ \\
\hline
\end{tabular}

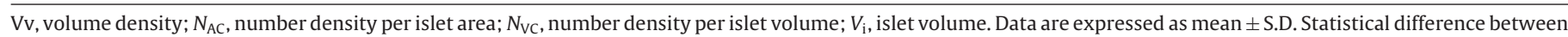
groups $\left({ }^{*} p<0.001\right)$. 

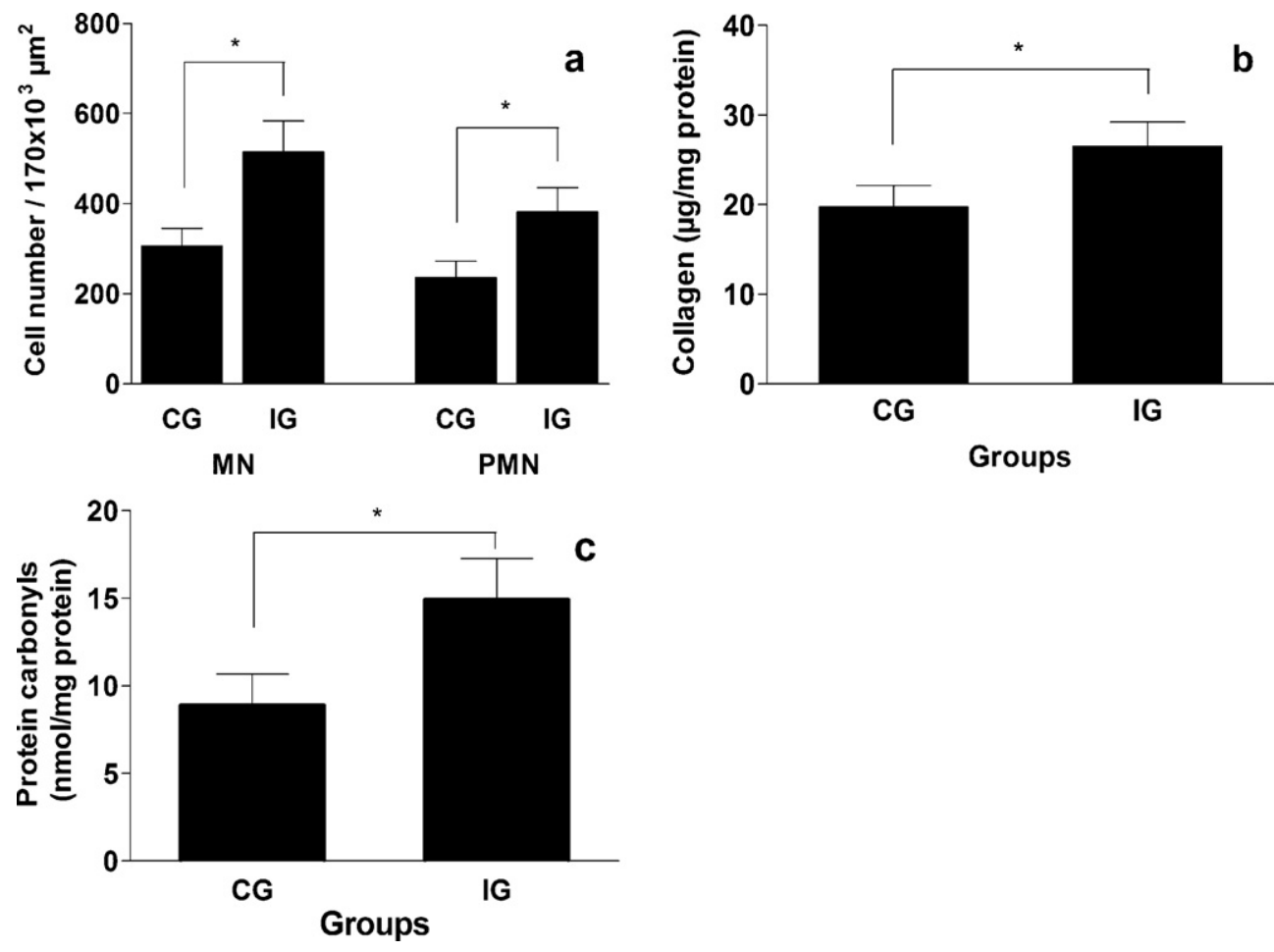

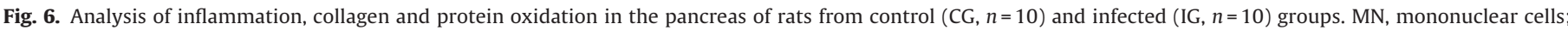
PMN, polymorphonuclear cells. The data are expressed as mean \pm SD. The statistical difference between the groups was * $p<0.001$.

seem to be related to the peripheral insulin resistance in $T$. cruzi infection, a finding that was reinforced by the similar glucose decay rates $\left(K_{\mathrm{ITT}}\right)$ found in both groups. Insulin resistance cannot be disregarded in the pathogenesis of glucose metabolism dysfunctions in ChD; however, it has been reported that insulin resistance appears to be mainly related to the comorbidities associated with $\mathrm{ChD}$, such as obesity and diabetes mellitus [18,35].

The present study showed for the first time that $T$. cruzi infection affects glucose and lactate metabolism during exercise, leading to a reduction in exercise tolerance. Exercise intolerance has traditionally been related to cardiac dysfunction in chagasic subjects $[10,16,17,20]$; however, the metabolic mechanisms associated with exercise intolerance remain poorly understood. It is believed that immune-mediated mechanisms and the increase in oxidative stress triggered by $T$. cruzi could be associated with exercise intolerance in ChD. In fact, there is evidence to show that the increased secretions of chemokine MCP-1 induced by T. cruzi are related to muscle weakness and reduced exercise capacity in chagasic patients [40]. In addition, previous studies showed that $T$. cruzi infection reduces the activity of antioxidant enzymes and stimulates the production of free radicals, events that cause a dysfunction in the cellular metabolism of energy via the uncoupling of several enzymatic complexes integrated within the electron transport chain $[13,41,42]$. However, whether or not these molecular changes are associated with dysfunctions in glucose and lactate metabolism due to T. cruzi infection warrants further investigation.

Although no statistical differences were found between the blood glucose and lactate levels at the end of the exercise test, reductions in time to fatigue and the distance traveled during the exercise test were found. In addition, the blood glucose and lactate levels showed an inverse and significant correlation at the end of the exercise test, similar to those observed between lactate and total time to fatigue. These findings suggest that the IG animals presented a higher energetic expenditure and a more active anaerobic metabolism compared to the CG. A lower energetic efficiency due to the increased anaerobic metabolism of glucose is proposed as a mechanism for partially explaining the exercise intolerance in $T$. cruzi infection. This mechanism is not unrealistic considering the possible negative influence of the infection in the electron transport chain and hence in the aerobic process of energy production $[13,41,42]$. In this context, a previous study showed that chagasic patients had increased levels of anaerobic metabolism that were associated with vascular dysfunction, reduced levels of $\mathrm{VO}_{2}$ max, lactate dehydrogenase, citrate synthase and, consequently, exercise tolerance [23]. Thus, a more glycolytic and less oxidative glucose metabolism is consistent with an increased production of lactate and with the anticipation of fatigue point during a progressive exercise protocol, as used in the present study. However, there is sufficient evidence that the determination of exercise tolerance is multifactorial. Thus, as $T$. cruzi is able to parasitize and damage structures such as peripheral nerves and skeletal muscles, equally important elements in determining the exercise tolerance $[16,17,23,40]$, we cannot attribute the results exclusively to the metabolic changes. In this context, the weak correlation between glucose and lactate levels and time to fatigue indicates that other organs and tissues should be investigated to improve the knowledge about the pathophysiological mechanism related to exercise intolerance in Chagas' disease.

Histopathological evidence of pancreatitis was observed in the IG animals. Based on the stereological analysis of the IG, morphological changes in the exocrine pancreas were more pronounced compared to those in the endocrine pancreas. However, the karyometric parameters of the $\beta$ cells in the IG animals were markedly reduced compared to the CG animals. From a morphological point of view, in the case of pancreatitis caused by T. cruzi, several other hypotheses can be used in an attempt to explain the dysfunction in glucose kinetics, such as hypoinsulinemia caused by insulitis, chronic inflammation with pancreatic fibrosis and microvascular damage, and parasympathetic denervation of pancreatic islets with the predominance of sympathetic stimuli $[7,26,34,35]$. 
Similar mechanisms have been described in other severe protozoan infections, such as malaria [6,34]. Some studies reported that hypoinsulinemia caused by the direct disruption of $\beta$ cells by $T$. cruzi may be an important mechanism involved in the dysfunction of glucose metabolism, especially hyperglycemia [18,34]. This mechanism was not supported in the present study because the number of $\beta$ cells in the pancreatic islets of IG animals compared to CG animals was not reduced. However, possible functional changes rather than structural defect in insulin-producing cells cannot be excluded. Thus, it is possible that the occurrence of hypoinsulinemia in T. cruzi infection was due to a reduction in the glucose-induced release of insulin by $\beta$ cells and a failure in the counter-regulation of hypoglycemia dependent on parasympathetic stimuli $[18,26,34]$. Furthermore, due to the high content of protein carbonyl in pancreatic tissue, we cannot rule out the possibility of an endocrine pancreatic dysfunction secondary to oxidative inhibition of receptors or other proteins that participate in cell signaling pathways. Although this mechanism is possible, it must still be proven. In fact, as an abnormal glucose curve in the OGTT was observed in the present study, it is possible that functional changes in $\beta$ cells could be directly linked to a dysfunction in glucose metabolism at rest rather than peripheral insulin resistance. As the levels of insulin were not investigated these functional changes cannot be determined, which was the main limitation of the present study.

Although many hypotheses have been proposed, the physiopathological mechanisms of glucose metabolism dysfunctions in ChD remain poorly understood. This situation is unfortunately worsened by the shortage of studies on the metabolic repercussions of $T$. cruzi infection related to organs other than the heart and gut. Thus, additional studies should be performed to better clarify the biochemical and molecular basis of glucose metabolism dysfunctions at rest and during exercise in $T$. cruzi infection. Taken together, the data obtained from this study allow us to conclude that $T$. cruzi infection impaired glucose metabolism at rest and during exercise in rats, which could constitute an additional mechanism underlying the exercise intolerance encountered in ChD. It is important to emphasize the fact that as these metabolic changes were observed in the absence of pronounced morphological changes of the pancreatic islets, further studies are required to better characterize the relationship between the endocrine pancreas and glucose metabolism at rest and during exercise in ChD. Finally, the results were important to indicate the need to extend investigations to other organs, suggesting that the pancreas may not be exclusively involved in the metabolic abnormalities observed in T. cruzi infection.

\section{Acknowledgments}

Research supported by FAPEMIG (PRONEX). Rômulo D. Novaes was recipient of Ph.D. scholarship from CAPES. Antonio J. Natali is a CNPq fellow.

\section{References}

[1] L.L. Bernardis, B.D. Petterson, Correlation between "Lee index" and carcass fat content in weanling and adult female rats with hypothalamic lesions, J. Endocrinol. 40 (1968) 527-528.

[2] M. Bradford, A rapid and sensitive method for quantitation of microgram quantities of protein utilizing the principle of protein-dye-binding, Anal. Biochem. 72 (1976) 248-254

[3] Z. Brener, Therapeutic activity and criterion of cure on mice experimentally infected with Trypanosoma cruzi, Rev. Inst. Med. Trop. São Paulo 4 (1962) 389-396.

[4] A. Biolo, A.L. Ribeiro, N. Clausell, Chagas cardiomyopathy-where do we stand after a hundred years? Prog. Cardiovasc. Dis. 52 (2010) 300-316.

[5] I.S. Caldas, A. Talvani, S. Caldas, C.M. Carneiro, M. Lana, P.M.M. Guedes, M.T. Bahia, Benznidazole therapy during acute phase of Chagas disease reduces parasite load but does not prevent chronic cardiac lesions, Parasitol. Res. 103 (2008) 413-421.
[6] I.A. Clark, F.M. Al Yaman, L.S. Jacobson, The biological basis of malarial disease, Int. J. Parasitol. 27 (1997) 1237-1249.

[7] C.E.P. Corbett, L.H.G. Scremin, R.A. Lombardi, J.J. Gama-Rodrigues, M. Okumura, Pancreatic lesions in acute experimental Chagas' disease, Rev. Hosp. Clin. Fac. Med. São Paulo 57 (2002) 63-66.

[8] W. Creutzfeldt, R. Ebert, M. Nauck, F. Stöckmann, Disturbances of the enteroinsular axis, Scand. J. Gastroenterol. 82 (1983) 111-119.

[9] H.A. Durham, G.E. Truett, Development of insulin resistance and hyperphagia in Zucker fatty rats, Am. J. Physiol. Regul. Integr. Comp. Physiol. 290 (2006) $652-658$.

[10] L. Gallo Jr., J.A. Neto, J.C. Manco, A. Rassi, D.S. Amorim, Abnormal heart rate responses during exercise in patients with Chagas' disease, Cardiology 60 (1975) 147-162.

[11] G. Gomori, Aldehyde fuchsin: a new stain for elastic tissue, Am. J. Clin. Pathol. 20 (1950) 665-666.

[12] H.J. Gundersen, T.F. Bendtsen, L. Korbo, N. Marcussen, A. Moller, K. Nielsen, J.R. Nyengaard, B. Pakkenberg, F.B. Sorensen, A. Verterby, Some new, simple and efficient stereological methods and their use in pathological research and diagnosis, APMIS 96 (1988) 379-394.

[13] S. Gupta, J. Wen, N.J. Garg, Oxidative stress in Chagas disease, Interdiscip. Perspect. Infect. Dis. (2009) 1-8 (ID190354).

[14] L.G. Koch, S.L. Britton, Artificial selection for intrinsic aerobic endurance running capacity in rats, Physiol. Genomics 5 (2001) 45-52.

[15] A.C. Lacerda, U. Marubayashi, C.H. Balthazar, C.C. Coimbra, Evidence that brain nitric oxide inhibition increases metabolic cost of exercise, reducing running performance in rats, Neurosci. Lett. 393 (2006) 260-263.

[16] M.M.O. Lima, M.C. Pereira, M.O.C. Rocha, F.R. Beloti, M.C.N. Alencar, A.L.P. Ribeiro, Left ventricular diastolic function and exercise capacity in patients with Chagas cardiomyopathy, Echocardiography 27 (2010) 519-524.

[17] M.M.O. Lima, M.O.C. Rocha, M.C.P. Nunes, L. Sousa, H.S. Costa, M.C.N. Alencar, R.R. Britto, A.L.P. Ribeiro, A randomized trial of the effects of exercise training in Chagas cardiomyopathy, Eur. J. Heart Fail. 12 (2010) 866-873.

[18] R.G. Long, R.H. Albuquerque, A. Prata, A.J. Barnes, T.E. Adrian, N.D. Christofides, S.R. Bloom, Response of plasma pancreatic and gastrointestinal hormones and growth hormone to oral and intravenous glucose and insulin hypoglycaemia in Chagas's disease, Gut 21 (1980) 772-777.

[19] A. López-De León, M. Rojkind, A simple micromethod for collagen and total protein determination in formalin-fixed paraffin-embedded sections, Histochem. Cytochem. 33 (1985) 737-743.

[20] C. Mady, B.M. Ianni, E. Arteaga, V.M.C. Salemi, C.C. Frimm, Maximal functional capacity in patients with Chagas' cardiomyopathy without congestive heart failure, J. Card. Fail. 3 (2000) 220-224.

[21] C.A. Mandarim-de-Lacerda, Stereological tools in biomedical research, An. Acad. Bras. Ciênc. 75 (2003) 469-486.

[22] P.M. Martinelli, E.R.S. Camargos, A.A. Azevedo, E. Chiari, G. Morel, C.R.S. Machado, Cardiac NGF and GDNF expression during Trypanosoma cruzi infection in rats, Auton. Neurosci. 130 (2006) 32-40.

[23] M. Montes de Oca, S.H. Torres, J.G. Loyo, F. Vazquez, N. Hernández, B. Anchustegui, J.J. Puigbó, Exercise performance and skeletal muscles in patients with advanced Chagas' disease, Chest 125 (2004) 1306-1314

[24] S.A. Morris, H.B. Tanowitz, M. Wittner, J.P. Bilezikian, Pathophysiological insights into the cardiomyopathy of Chagas' disease, Circulation 82 (1990) 1900-1909.

[25] R.D. Novaes, R.V. Gonçalves, D.C. Marques, M.C. Cupertino, M.C. Peluzio, J.P. Leite, I.R. Maldonado, Effect of bark extract of Bathysa cuspidata on hepatic oxidative damage and blood glucose kinetics in rats exposed to paraquat, Toxicol. Pathol. 40 (2012) 62-70.

[26] L.C.M. Oliveira, Y. Juliano, N.F. Novo, M.M. Neves, Blood glucose and insulin response to intravenous glucose by patients with chronic Chagas' disease and alcoholism, Braz. J. Med. Biol. Res. 26 (1993) 1187-1190.

[27] P. Pushparaj, C.H. Tan, B.K.H. Tan, Effects of Averrhoa bilimbi leaf extract on blood glucose and lipids in streptozotocin-diabetic rats, J. Ethnopharmacol. 72 (2000) 69-76.

[28] A. Rassi-Jr, A. Rassi, J.A. Marin-Neto, Chagas disease, Lancet 375 (2010) 1388-1402.

[29] P.G. Reeves, F.H. Nielsen, G.C. Fahey Jr., AIN-93 purified diets for laboratory rodents: final report of the American Institute of Nutrition ad hoc writing committee on the reformulation of the AIN-76A rodent diet, J. Nutr. 123 (1993) 1939-1951.

[30] A. Rocha, L.C. de Oliveira, R.S. Alves, E.R. Lopes, Pancreatic neuronal loss in chronic Chagas' disease patients, Rev. Soc. Bras. Med. Trop. 31 (1998) 43-49.

[31] M.A. Sala, M.C. Komesu, R.A. Lopes, C.G. Maia, Karyometric study of basal cell carcinoma, Braz. Dent. J. 5 (1994) 11-14.

[32] J.C. Saldanha, V.M. Santos, M.A. Reis, D.F. Cunha, V.P.A. Teixeira, Morphologic and morphometric evaluation of pancreatic islets in chronic Chagas' disease, Rev. Hosp. Clin. Fac. Med. Sao Paulo 56 (2001) 131-138.

[33] V.M. Santos, M.A. Lima, M. Cabrine-Santos, D.E. Márquez, M.G. Reis, G.A. Pereira, E. Lages-Silva, L.E. Ramírez, Pancreatic hepatocytes in hamsters (Mesocricetus auratus) infected with Trypanosoma cruzi, Exp. Parasitol. 100 (2002) 103-111.

[34] V.M. Santos, M.A. Lima, M. Cabrine-Santos, D.S. Marquez, G.A. Pereira, E. LagesSilva, L.E. Ramírez, Functional and histopathological study of the pancreas in hamsters (Mesocricetus auratus) infected and reinfected with Trypanosoma cruzi, Parasitol. Res. 94 (2004) 125-133.

[35] V.M. Santos, S.F.C. Cunha, V.P. Teixeira, J.P. Monteiro, J.A. Santos, T.A. Santos, L.A Santos, D.F. Cunha, Frequency of diabetes mellitus and hyperglycemia in chagasic and non chagasic women, Rev. Soc. Bras. Med. Trop. 32 (1999) 489-496. 
[36] C. Schebeleski-Soares, R.C. Occhi, S.M. Franzói-de-Moraes, M.M.D. Oliveira, F.N. Almeida, M.J.O. Toledo, S.M. Araújo, Preinfection aerobic treadmill training improves resistance against Trypanosoma cruzi infection in mice, Appl. Physiol. Nutr. Metab. 34 (2009) 1-8.

[37] W.M. Sherman, Metabolism of sugars and physical performance, Am. J. Clin. Nutr. 62 (1995) 228S-241S.

[38] R.S. Sohal, S. Agarwal, A. Dubey, W.C. Orr, Protein oxidative damage is associated with life expectancy of houseflies, Proc. Natl. Acad. Sci. 90 (1993) 7255-7259.

[39] L.A. Sternberger, The unlabelled antibody peroxidase antiperoxidase (PAP) method, in: S. Cohen, R.T. McClusky (Eds.), Immunocytochemistry, second ed. John Wiley and Sons, New York, 1979, pp. 104-169.
[40] L. Sousa, F.A. Botoni, R.R. Britto, M.O.C. Rocha, A.L. Teixeira-Jr, M.M. Teixeira, A.M. Reis, B.M.R. Oliveira, A.L. Ribeiro, Six-minute walk test in Chagas cardiomyopathy, Int. J. Cardiol. 125 (2008) 139-141.

[41] J. Wen, M. Dhiman, E.B. Whorton, N.J. Garg, Tissue-specific oxidative imbalance and mitochondrial dysfunction during Trypanosoma cruzi infection in mice, Microbes Infect. 10 (2008) 1201-1209.

[42] J. Wen, N. Garg, Oxidative modification of mitochondrial respiratory complexes in response to the stress of Trypanosoma cruzi infection, Free Radic. Biol. Med. 37 (2004) 2072-2081. 